\title{
AVALIAÇÃO DO EQUILÍBRIO CORPORAL E DA FORÇA ISOCINÉTICA DE FLEXORES E EXTENSORES DE JOELHO DE UM IDOSO SARCOPÊNICO, DIABÉTICO COM DEFICIÊNCIA VISUAL TOTAL: ESTUDO DE CASO COMPARATIVO
}

\author{
BODY BALANCE AND ISOKINETIC STRENGTH ASSESSMENT OF THE FLEXORS AND KNEE EXTEN- \\ SORS IN A SARCOPENIC, DIABETIC ELDERLY INDIVIDUAL WITH COMPLETE VISUAL IMPAIRMENT: \\ A COMPARATIVE CASE STUDY
}

\section{Daniela Virote Kassick Müller ${ }^{a^{*}}$, Graziela Morgana Silva Tavares ${ }^{b^{*}}$, Maria Gabriela Valle Gottlieb ${ }^{\mathrm{c}^{* *}}$, Rodolfo Herberto Schneider ${ }^{\mathrm{d}^{* *}}$}

\author{
adanivkm@gmail.com, bgrazielatavares@unipampa.edu.br, cmaria.gottlieb@pucrs.br, drodolfo.schneider@pucrs.br \\ *Universidade Federal do Pampa - Uruguaiana (RS), Brasil \\ **Pontifícia Universidade Católica do Rio Grande do Sul - Porto Alegre (RS), Brasil
}

Data de recebimento do artigo: 29/09/2015

Data de aceite do artigo: 25/02/2016

\section{RESUMO}

Objetivo: Avaliar, descrever e comparar o equilíbrio corporal e a força muscular isocinética de flexores e extensores de joelho de um idoso sarcopênico, com diabetes mellitus tipo 2 (DM2) e deficiência visual total, com dois idosos com características semelhantes; porém, sem deficiência visual. Descriçáo do caso: Este estudo descritivo-comparativo analisou o caso de um idoso sarcopênico, com DM2 e deficiência visual total (sujeito 1), e o comparou com dois idosos sarcopênicos, com DM2, porém, sem deficiência visual (sujeitos 2 e 3). Foram avaliadas variáveis de equilíbrio pelo Sensory Organization Test (SOT), utilizando o Smart Equitest ${ }^{\mathrm{Tm}} \mathrm{da}$ NeuroCom ${ }^{\oplus}$ International em seis diferentes condiçóes nas quais foram solicitados os mecanismos de controle postural de diferentes formas. Para cada condição foram realizadas três tentativas, gerando ao final uma média do desempenho para cada condição. Posteriormente foi realizada a aquisição de dados sobre força e potência muscular dos flexores e extensores do joelho através do dinamômetro isocinético Biodex System 4 Pro nas velocidades de 60 e 180\% $/ \mathrm{seg}$. Conclusáo: Os resultados mostraram que apesar da deficiência visual total o sujeito 1 apresentou um bom equilíbrio quando comparado com a média geral e com os sujeitos 2 e 3, mas apresentou um déficit de força muscular maior que os demais.

Palavras-chave: Idoso; equilíbrio postural; deficiência visual; sarcopenia.

\section{ABSTRACT}

Objective: assess, describe and compare the body balance and the muscle strength of knee flexors and extensors of a sarcopenic elderly individual with type 2 Diabletes Mellitus (DM2) and total visual impairment, with two other elderlies in similar conditions, but without visual impairment. Case description: This descriptive-comparative study assessed a sarcopenic elderly patient with DM2 and total visual impairment (1 subject), comparing him with 2 sarcopenics elderly patients with DM2, but without visual impairment (subjects 2 and 3). Balance variables were evaluated by the Sensory Organization Test (SOT), using NeuroCom ${ }^{\circ}$ International Smart Equitest ${ }^{\mathrm{TM}}$ in six different conditions in which postural control mechanisms were requested in different ways. For each condition three attempts were performed, generating then a performance average for each condition. It was later gathered data on knee flexors and extensors muscle strength and power by Biodex System $4 \mathrm{Pro}^{\circ}$ isokinetic dynamometer at speeds of 60 and $180^{\circ}$ sec. Conclusion: The study results showed that the subject 1 even presenting total visual impairment presented a good body balance when compared with the overall average, and with subjects 2 and 3, but presented a greater muscle strength deficit in comparison with subjects 2 and 3 .

Keywords: Elderly; postural balance; visual impairment; sarcopenia. 


\section{Introdução}

O tecido musculoesquelético é o maior tecido do corpo humano, compreendendo também o maior componente proteico do organismo. Os músculos não só são responsáveis pela autonomia motora dos indivíduos, como participam da homeostase glicêmica e metabólica ${ }^{1}$. Dessa forma, a massa muscular será resultado do equilíbrio entre a produção e o catabolismo de suas proteínas.

Apesar de o catabolismo proteico ser um processo natural, vários fatores podem aumentar essa condição destrutiva, favorecendo o desequilíbrio e, consequentemente, diminuição de massa muscular. Alguns dos fatores que levam ao catabolismo proteico incluem a resistência à insulina e níveis elevados de glicocorticoides, diminuição da condutibilidade nervosa, reaçōes inflamatórias, desuso, restrição calórica, estresse oxidativo dentre outros. No entanto, quando ocorre essa diminuição da massa muscular, além do comprometimento da força, potência e do equilíbrio corporal, observa-se ainda mais resistência insulínica, o que pode levar ou até mesmo agravar a diabetes mellitus tipo 2, hiperadiposidade, menor reparo tecidual e insuficiência do sistema imunitário ${ }^{1,2}$.

Uma persistente hiperglicemia decorrente da falta de insulina ou à resistência do organismo a esse hormônio desencadeia a diabetes mellitus, que pode ocasionar tanto complicaçóes agudas como tardias, sendo que as últimas provocam elevada morbidade e mortalidade, como é o caso da retinopatia e/ou maculopatia diabética, uma causa importante de cegueira ${ }^{3}$.

Contudo, é importante destacar que a perda de massa muscular é uma das modificações da composição corporal que estão intimamente relacionadas ao envelhecimento, ou seja, com o avanço da idade é natural que haja perda de massa muscular e um aumento de massa gorda. No entanto, se essas modificaçóes estiverem associadas à perda de força muscular e/ou funcionalidade, pode-se caracterizar como sarcopenia ${ }^{4}$.

A sarcopenia constitui-se de uma entidade sindrômica de causa multifatorial, definida por Baumgartner et al. ${ }^{5}$, como sendo a diminuição de massa muscular esquelética dos desvios-padróes abaixo da média do grupo controle constituído por indivíduos jovens com idade de 29 anos, saudáveis, pareados para a mesma etnia. A fim de melhorar o rastreamento dessa desordem, Cruz-Jentoft et al. ${ }^{6}$ elaboraram um algoritmo que engloba diversos aspectos do indivíduo idoso, como velocidade da marcha, independência funcional, força e massa muscular para o possível diagnóstico de sarcopenia. Sobretudo, a sarcopenia é uma das condiçóes mais frequentes que acomete o sistema musculoesquelético de idosos.

Um dos principais fatores que levam a sarcopenia relacionada ao envelhecimento é a falta de atividade física. Estudos têm mostrado que idosos sedentários apresentam menor massa muscular e, com isso, maior prevalência de incapacidade funcional. A diminuiçáo da força muscular, potência e resistência apresentada em idosos sarcopênicos contribuem no comprometimento do equilíbrio corporal. Essa situação pode favorecer o risco de quedas e, como desfecho, a possibilidade de fraturas e suas consequências, como incapacidade, dependência, hospitalização e até mesmo o óbito ${ }^{7-9}$.

Equilíbrio, balanço e controle postural sáo apresentados como sinônimos para conceituar o mecanismo pelo qual o corpo se protege de quedas ao recuperar o centro de massa corporal sobre a base de sustentação, controlando a posição do corpo no espaço ${ }^{10}$. Para manter esse controle, é necessário um equilíbrio de açóes entre os sistemas somatossensorial, visual e vestibular em conjunto com o aparelho locomotor e da integração de suas atividades no sistema nervoso central ${ }^{11}$.

São estimados que a prevalência de queixas de falta de equilíbrio na população acima de 65 anos chega a $85 \%$ e que as fraturas decorrentes de quedas são responsáveis por aproximadamente $70 \%$ das mortes acidentais em pessoas com mais de 75 anos $^{12}$. Esses achados ocorrem pela degeneração dos sistemas responsáveis pelo equilíbrio, como é o caso da diminuição da acuidade visual e dos reflexos por eles gerados ${ }^{13,14}$, além da diminuição da força em consequência da sarcopenia no envelhecimento ${ }^{15,16}$. A visão é um processo complexo que envolve a integração das estruturas do olho, o controle motor e o controle neural e sua importância está em captar informaçóes sobre o ambiente e na orientação do movimento do corpo. Quando esta se encontra diminuída ou ausente, a prevalência de desequilíbrio e quedas tende a aumentar ${ }^{14}$.

No entanto, quanto maior o número de fatores de risco e comorbidades presentes no indivíduo idoso, maior será a chance de desequilíbrio e, com isso, a possibilidade de queda e fratura. Diante do exposto, este estudo realizou uma avaliação do equilíbrio corporal e da força muscular de flexores e extensores do joelho de um idoso com diagnóstico de sarcopenia e deficiência visual total, comparando seus resultados com dois sujeitos sarcopênicos e diabéticos, mas sem deficiência visual.

\section{Descrição dos casos}

Este estudo foi aprovado pelo Comitê de Ética em Pesquisa da Pontifícia Universidade Católica do Rio Grande do Sul (PUC-RS), seguindo as orientaçôes e diretrizes da Comissão Nacional de Ética em Pesquisa (CONEP) (protocolos 312.127 e 930.945/15). Os indivíduos foram informados sobre a importância da pesquisa através do Termo de Consentimento Livre e Esclarecido (TCLE), assinando esse documento ao final da explicação. 
O estudo em questão foi composto por três indivíduos do sexo masculino, o sujeito 1, o estudo de caso, e os sujeitos 2 e 3 , idosos com características semelhantes; porém, sem deficiência visual a fim de comparação. A média de idade dos três sujeitos foi de 77,7 anos. Os três sujeitos foram questionados sobre diagnósticos de doenças prévias, histórico de quedas no último ano, histórico prévio de realizaçáo do exame de densitometria óssea, hábitos de vida, tais como: prática de atividade física, ingestão de bebidas alcóolicas, tabagismo dentre outros. Posteriormente, foram adquiridas as medidas antropométricas (massa, estatura, circunferências e dobras cutâneas) para definir o índice de massa muscular (IMM) $)^{7,17,18}$.

Em sequência, foram realizadas a avaliação do equilíbrio corporal e a avaliação isocinética de força muscular dos flexores e extensores da articulação do joelho. A primeira foi avaliada através do equipamento Balance Manager, da NeuroCom ${ }^{\circledast}$ International. O teste foi realizado da seguinte forma: a) cada idoso foi instruído a retirar os sapatos e se colocar em ortostase, com apoio bipodal, sobre as marcaçôes da plataforma de frente para o monitor; b) cada idoso foi vestido com o colete de segurança regulado de acordo com suas dimensôes individuais para evitar qualquer risco de queda; c) os sujeitos foram informados que haveria uma sequência de seis testes para avaliaçáo do equilíbrio corporal; cada um deles em diferentes situaçóes segundo orientaçóes do fabricante, tais como: olhos abertos e fechados, plataforma fixa ou móvel, cabine fixa ou móvel. Caso o idoso fizesse o uso de lentes corretivas, ele seria solicitado a utilizá-las durante a avaliação nas condiçóes que necessitassem os olhos abertos; já na condição de olhos fechados, foi fornecido aos indivíduos uma venda para que não houvesse qualquer resquício de luminosidade durante o teste; d) cada etapa do teste apresentou três repetições com duração de 20 segundos cada (Figura 1).

Figura 1: As seis condições realizadas no Sensory Organization Test (SOT).
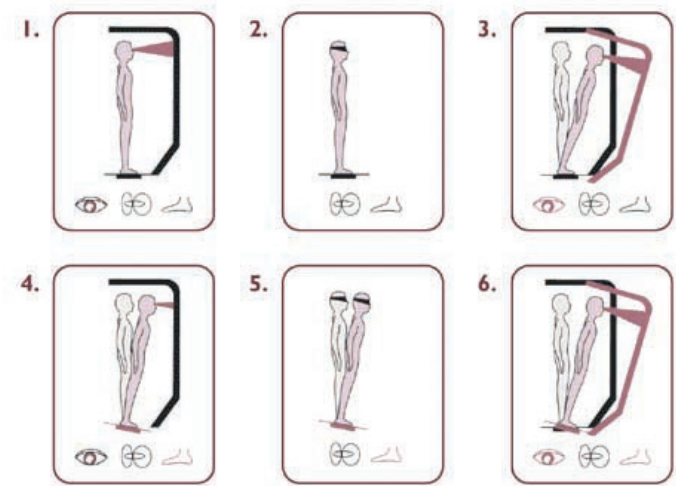

Sensory Organization Test

Fonte: NeuroCom International (2007)
Após a realização do teste, os indivíduos tiveram um intervalo de 15 minutos para descansar. A segunda etapa da avaliação constou da aquisiçâao de dados sobre força e potência muscular dos membros inferiores através do dinamômetro isocinético Biodex System 4 Pro, sendo realizada como se segue: a) foi mensurada a força e potência muscular dos flexores e extensores do joelho, tanto no lado direito como no lado esquerdo; b) o teste foi realizado com o encosto da cadeira inclinado a $85^{\circ}$ e o eixo rotacional do aparelho (dinamômetro) alinhado com o côndilo lateral do fêmur, com a almofada da alavanca posicionada $3 \mathrm{~cm}$ acima do maléolo lateral, e a amplitude de movimento (ADM) testada foi a máxima conseguida pelo paciente sem desconforto para cada membro inferior ${ }^{19}$; c) os indivíduos idosos executaram para cada membro três séries de exercícios: a primeira série foi executada para que pudesse aprender o gesto motor e o funcionamento do equipamento (trial), a segunda teve o objetivo de avaliar a força máxima (pico de torque) para o membro analisado e a terceira analisou o pico de torque, a potência e relaçáo de tempo de fadiga para a musculatura estudada. Cada série utilizou contraçóes concêntricas, velocidades angulares constantes e predeterminadas, a primeira (trial) e a segunda de $60 \%$ s ( 5 repetiçóes) e a terceira de $180 \%$ s (10 repetiçóes) para o joelho. Antes de iniciar uma nova série, o idoso teve um intervalo de 90 segundos para que pudesse se recuperar do exercício. As coletas foram iniciadas sempre pelo membro inferior direito e após foi repetida no membro inferior esquerdo.

Os dados deste estudo foram analisados de forma descritiva.

\section{Resultados}

$\mathrm{Na}$ Tabela 1 são apresentados os resultados referentes aos dados antropométricos dos indivíduos do estudo de acordo com os cálculos do índice de massa muscular (IMM) que classifica os sujeitos com sarcopenia $\left(\mathrm{IMM}<10,75 \mathrm{~kg} / \mathrm{m}^{2}\right)$.

Tabela 1: Características antropométricas dos sujeitos avaliados.

\begin{tabular}{|c|c|c|c|c|c|c|}
\hline & $\begin{array}{l}\text { Idade } \\
\text { (anos) }\end{array}$ & $\begin{array}{c}\text { Massa } \\
(\mathrm{kg})\end{array}$ & $\begin{array}{c}\text { Estatura } \\
\text { (m) }\end{array}$ & $\begin{array}{c}\text { IMC } \\
\left(\mathrm{kg} / \mathrm{m}^{2}\right)\end{array}$ & MM & $\begin{array}{c}\text { IMM } \\
\left(\mathrm{kg} / \mathrm{m}^{2}\right)\end{array}$ \\
\hline Sujeito 1 & 77 & 66.5 & 1.54 & 28.04 & 18.879 & 7.960 \\
\hline Sujeito 2 & 78 & 70.4 & 1.68 & 24.94 & 26.904 & 9.532 \\
\hline Sujeito 3 & 78 & 73.5 & 1.69 & 25.73 & 25.861 & 9.055 \\
\hline
\end{tabular}


Ao serem questionados sobre o histórico de doenças prévias, o sujeito 1 relatou possuir diabetes mellitus do tipo 2 (DM2), controlando-a por meio do uso de medicamentos, e deficiência visual total sem presença de percepçáo luminosa por retinopatia diabética bilateral há seis anos. Os sujeitos 2 e 3 também relataram história prévia de DM2; no entanto, o sujeito 2 negou qualquer tipo de alteraçáo visual, e o sujeito 3 fazia uso de lentes corretivas apenas para melhora da acuidade visual diminuída pelo processo de envelhecimento. Todos os sujeitos negaram a presença de outras comorbidades e classificaram o estado de saúde geral como regular.

Em relaçáo aos hábitos de vida, todos os sujeitos negaram realizar o consumo de bebidas alcóolicas; entretanto, referiram o uso de tabaco no passado, tendo o sujeito 1 parado há seis anos, o sujeito 2 há mais de vinte anos e o sujeito 3 entre 10 e 20 anos. $\mathrm{O}$ consumo médio do sujeito 1 era de 31 a 40 cigarros por dia, e o dos sujeitos 2 e 3 era de 11 a 20 cigarros por dia. Quando questionados sobre a prática de atividade física, os sujeitos 2 e 3 relataram que realizam caminhadas e ginástica há cerca de um ano, ambos com uma frequência de 2 vezes por semana. Já o sujeito 1 relatou náo realizar atividades físicas pela deficiência visual, mas anteriormente ao ocorrido também era sedentário. Além disso, ele não foi orientado quanto a qualquer processo de reabilitação ou readequação após a perda da visão para realizar suas atividades de vida diária. Para deambular, apenas o sujeito 1 faz uso de dispositivo auxiliar, sendo este do tipo andador ou mesmo bengala convencional, não utilizando a bengala branca para sua locomoção e orientação.

Os sujeitos 1 e 2 não apresentavam queixas vestibulares, tais como vertigem ou tontura, ao contrário do sujeito 3 que apresentava ambas. Mesmo assim, os sujeitos 1 e 2 apresentaram dois episódios de queda no último ano, e o sujeito 3 apresentou mais de 3 episódios de quedas nesse mesmo período. Nenhum dos três sujeitos realizou qualquer exame complementar para avaliar a densidade mineral óssea, como densitometria, e negam ter tido algum tipo de fratura durante as quedas. No entanto, todos referiram possuir medo e ansiedade de cair. Os resultados da avaliaçáo do equilíbrio corporal são apresentados na Tabela 2.

Os resultados da avaliação da força muscular de membros inferiores nas articulaçóes dos joelhos em velocidade de 60 e 180\%/seg são apresentados na Tabela 3 e, respectivamente, nas figuras 2 e 3 .
Tabela 2: Média percentual do equilíbrio corporal em diferentes condições do Sensory Organization Test (SOT).

\begin{tabular}{|c|c|c|}
\hline Condição & & Média (\%) \\
\hline \multirow{3}{*}{$\begin{array}{l}\text { Plataforma fixa, cabine fixa e } \\
\text { olhos abertos }\end{array}$} & sujeito 1 & 90,33 \\
\hline & sujeito 2 & 93.66 \\
\hline & sujeito 3 & 87,33 \\
\hline \multirow{3}{*}{$\begin{array}{l}\text { Plataforma fixa, cabine fixa e } \\
\text { olhos fechados }\end{array}$} & sujeito 1 & 90,66 \\
\hline & sujeito 2 & 91,33 \\
\hline & sujeito 3 & 78 \\
\hline \multirow{3}{*}{$\begin{array}{l}\text { Plataforma fixa, cabine móvel e } \\
\text { olhos abertos }\end{array}$} & sujeito 1 & 89 \\
\hline & sujeito 2 & 91,33 \\
\hline & sujeito 3 & 77,33 \\
\hline \multirow{3}{*}{$\begin{array}{l}\text { Plataforma móvel, cabine fixa e } \\
\text { olhos abertos }\end{array}$} & sujeito 1 & 88,66 \\
\hline & sujeito 2 & 87,33 \\
\hline & sujeito 3 & 46,66 \\
\hline \multirow{3}{*}{$\begin{array}{l}\text { Plataforma móvel, cabine fixa e } \\
\text { olhos fechados }\end{array}$} & sujeito 1 & 48,33 \\
\hline & sujeito 2 & 68 \\
\hline & sujeito 3 & 37,66 \\
\hline \multirow{3}{*}{$\begin{array}{l}\text { Plataforma móvel, cabine móvel e } \\
\text { olhos abertos }\end{array}$} & sujeito 1 & 49,33 \\
\hline & sujeito 2 & 71 \\
\hline & sujeito 3 & 35 \\
\hline \multirow{3}{*}{ Média geral } & sujeito 1 & 76,05 \\
\hline & sujeito 2 & 81 \\
\hline & sujeito 3 & 59 \\
\hline
\end{tabular}

Tabela 3: Movimento realizado na articulação do joelho nas velocidades 60 e $180 \%$ seg.

\begin{tabular}{cccccc} 
& & $\begin{array}{c}\text { Extensáo } \\
\text { MID }\end{array}$ & $\begin{array}{c}\text { Flexáo } \\
\text { MID }\end{array}$ & $\begin{array}{c}\text { Extensáo } \\
\text { MIE }\end{array}$ & $\begin{array}{c}\text { Flexáo } \\
\text { MIE }\end{array}$ \\
\hline $\begin{array}{c}\text { Pico de } \\
\text { torque } \\
\mathbf{6 0} / \mathbf{s e g}\end{array}$ & sujeito 1 & 22,7 & 3,6 & 12,8 & 11,5 \\
\hline $\begin{array}{c}\text { Pico de } \\
\text { torque }\end{array}$ & sujeito 2 & 46,63 & 12,74 & 74,43 & 29,14 \\
$\mathbf{1 8 0} / \mathbf{s e g}$ & sujeito 1 & 44,74 & 8,94 & 31,31 & 64 \\
\hline $\begin{array}{l}\text { Trabalho } \\
\text { total }(\mathbf{J})\end{array}$ & sujeito 2 & 31,31 & 32,13 & 55,72 & 23,18 \\
$\mathbf{1 8 0} / \mathbf{s e g}$ & sujeito 1 & 135,8 & 10 & 61,4 & 0,7 \\
\hline & sujeito 2 & 167,44 & 22,5 & 356,71 & 20,6 \\
\hline & & & 13,15 & 30,64 & 13,01 \\
\hline
\end{tabular}


Figura 2: Movimento de extensão (away) e flexão (toward) da articulação do joelho em velocidade angular de $60 \%$ seg.
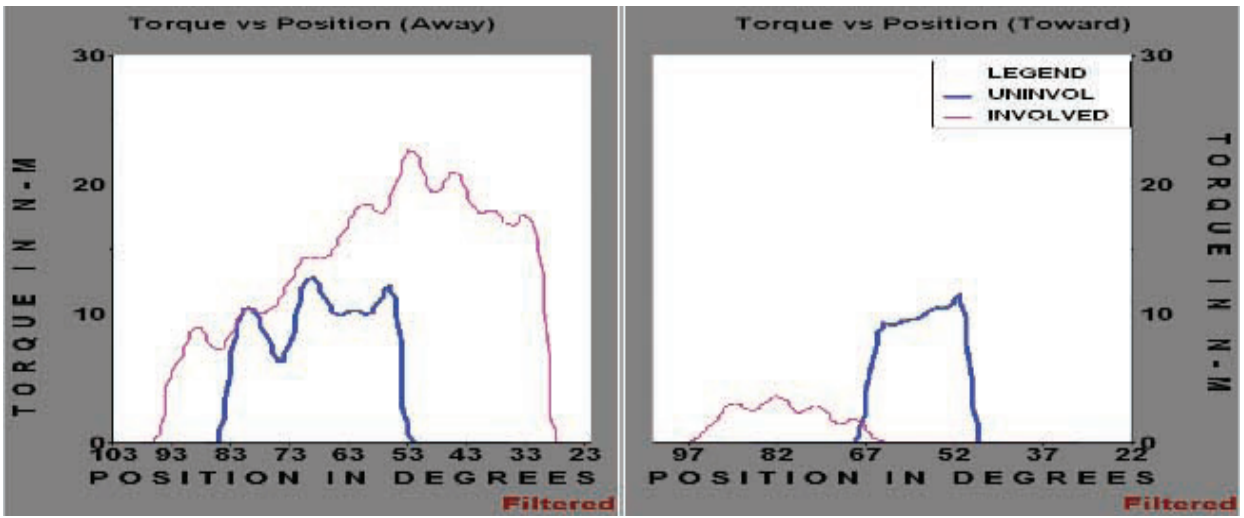

Legenda: Azul: uninvolved (membro inferior esquerdo: MIE); Rosa: involved (membro inferior direito: MID).

Figura 3: Movimento de extensão (away) e flexão (toward) da articulação do joelho em velocidade angular de $180^{\circ} / \mathrm{seg}$.
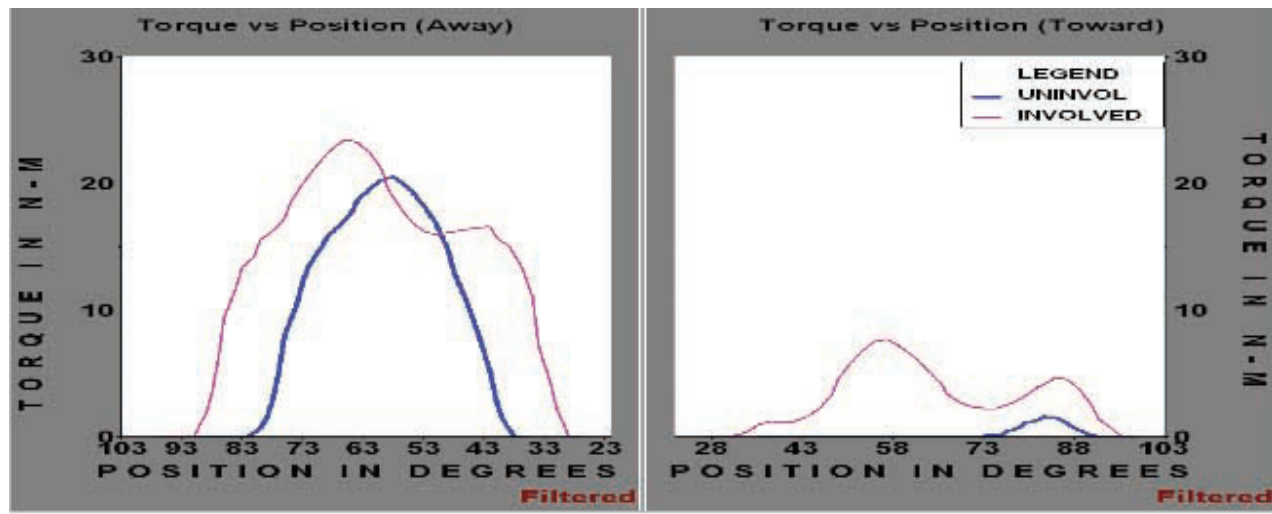

Legenda: Azul: uninvolved (membro inferior esquerdo: MIE); Rosa: involved (membro inferior direito: MID).

\section{Discussão}

No sistema musculoesquelético durante o processo de envelhecimento, é comum a perda da massa e força muscular. De acordo com Baumgartner et al. ${ }^{5}$, a sarcopenia acomete cerca de $30 \%$ dos indivíduos com idade acima de 60 anos, e esse percentual tende a aumentar com os anos, atingindo mais de $50 \%$ dos indivíduos com idade acima de 80 anos, com uma prevalência maior no sexo masculino do que no feminino $(26,8 \%$ e $22,6 \%$, respectivamente $)^{20}$.

Os resultados deste estudo evidenciaram que os três sujeitos avaliados apresentavam sarcopenia de acordo com a classificação antropométrica através do IMM; além disso, todos relataram possuir diabetes mellitus tipo 2 .

Estudos $^{21-23}$ têm demonstrado que existe correlação positiva entre a resistência à insulina e a manutenção de massa muscular, podendo um ser causa ou consequência do outro. A alteração da diabetes mellitus leva não só à perturbação do metabolismo glicídico como também dos lipídios e proteínas. A forma mais comum de apresentação da diabetes mellitus é a do tipo 2 , correspondendo a cerca de 90 a 95\% das situaçóes de diabetes. Como mencionado anteriormente, das complicaçôes tardias, a retinopatia e/ou maculopatia diabética são as principais causas de deficiência visual ${ }^{3}$. $\mathrm{O}$ tratamento da diabetes deve objetivar o bem-estar e a prevenção ou minimização dessas complicaçóes, que podem levar ao comprometimento da capacidade funcional dos pacientes ou até mesmo ao óbito.

Neste estudo, conforme evidenciado na Tabela 3, pode-se observar que o sujeito 1 apresenta menor força e potência muscular que os demais. Esses resultados são observados a partir da análise do pico de torque e do trabalho total exercido pelos flexores e extensores do joelho que se apresentam com valores menores quando comparados aos demais sujeitos. Isso ocorreu tanto na velocidade de $60 \%$ seg como em $180 \% / \mathrm{seg}$ em ambos os membros inferiores. Esse fato pode ter ocorrido devido a maior deficiência visual apresentada pelo sujeito 1 , que provoca maior imobilidade e, com isso, mais desuso da musculatura, tendendo a diminuir mais ainda a massa muscular, força e potência. Além disso, os sujeitos 2 e 3 relataram praticar atividade física há um ano, enquanto o primeiro relatou ser sedentário, mesmo antes da deficiência visual.

A perda da força muscular geralmente é iniciada a partir dos 25 a 30 anos de idade e ocorre devido a vários fatores ${ }^{12}$. 
No entanto, a diminuição da força muscular, potência e resistência da musculatura em idosos tende a ser mais intensa e pode afetar o equilíbrio e o desempenho funcional, dificultando inclusive atividades da rotina diária. Isso ocorre porque a diminuição gradual e crescente da massa muscular vai fazendo que o idoso adquira uma menor mobilidade, agilidade e funcionalidade, favorecendo o risco de quedas pela perturbação do equilíbrio e interferindo na independência desses indivíduos mais velhos ${ }^{12,24}$.

O controle postural é a capacidade de manter o equilíbrio oscilando ou recuperando o centro de massa corporal sobre a base de sustentaçáo, fazendo que nos mantenhamos em ortostase ou nos locomovamos com segurança apesar das perturbações externas ${ }^{25}$. Esse equilíbrio ou controle postural é determinado tanto pelas aferências sensoriais como pela integridade do aparelho locomotor e sistema musculoesquelético ${ }^{26,27}$. Além disso, esses sistemas devem estar perfeitamente integrados ao sistema nervoso central ${ }^{28}$, conexão que confere os reflexos responsáveis pela manutenção do equilíbrio corporal $^{29}$. O sistema visual fornece uma das principais aferências sensoriais para auxiliar na manutenção do equilíbrio corporal; quando este se encontra alterado ou ausente, espera-se que o controle postural seja afetado drasticamente ${ }^{15}$.

Os dados das variáveis do equilíbrio dos sujeitos deste estudo mostraram que o sujeito 1 , apesar da deficiência visual, conseguiu manter o controle postural com uma média geral de $76,05 \%$, maior que a de indivíduos saudáveis com a mesma faixa etária, que seria em torno de $65 \%$ de acordo com dados fornecidos pelo Neurocon ou quando comparado com o sujeito 3 deste estudo que obteve uma média geral de 59\%. No entanto, apresentou menor média quando comparado ao sujeito 2 , que apresentou uma média geral de $81 \%$.

Diversas teorias já foram elaboradas na tentativa de elucidar como a ausência visual pode ser compensada em indivíduos com alteração e/ou ausência visual, para manutenção do controle postural, sendo uma delas a da neuroplasticidade, que é conhecida como a capacidade do sistema nervoso de se reorganizar. Apesar de estar diminuída no envelhecimento, essa condição não está ausente e faz que o idoso na ausência da acuidade visual consiga estabelecer novas conexóes neurais que repercutam na melhora funcional do seu equilíbrio ${ }^{30}$. Outra teoria seria a de que outros sistemas seriam recrutados na tentativa de compensar o sistema visual. Em artigo de revisão realizado por Qin e $\mathrm{Yu}^{31}$ refere que o processamento cross-modal de sinais não visuais em áreas occipitais em sujeitos dotados de visão e em sujeitos cegos tardios sugere que a organização funcional do córtex visual é de natureza supramodal. Além disso, partes do córtex visual podem ser recrutadas por outras modalidades sensoriais para processar a informação sensorial de maneira compensatória ${ }^{32}$.
$\mathrm{Na}$ avaliação do equilíbrio deste estudo foram testadas seis condiçóes diferentes, nas quais o grau de dificuldade para manter o controle postural era crescente. Pôde-se observar (Tabela 2) que todos os indivíduos foram piorando sua performance com o aumento do nível de dificuldade da condição testada, sendo que, ao analisarmos separadamente os sistemas testados, observa-se que o sujeito 1 apresentou apenas um sistema com déficit, o sujeito 2 apresentou todos os sistema dentro dos parâmetros de normalidade e já o sujeito 3 apresentou déficit em dois dos três sistemas avaliados, visual e vestibular, sobrecarregando o sistema somatossensorial e, com isso, resultando em um desempenho na média geral do equilíbrio abaixo dos $65 \%$ recomendados para a faixa etária. Esse fato associado às queixas de síndrome vestibular dele talvez justifique a maior ocorrência de desequilíbrio e quedas.

Liaw et al. ${ }^{33}$ compararam as características do equilíbrio postural de 107 indivíduos saudáveis entre $16 \mathrm{e}$ 80 anos e mostraram que a oscilação corporal aumenta com a idade, o que pode interferir no equilíbrio; logo, a propensão a quedas se torna cada vez maior. Quando há uma perturbação no equilíbrio, a queda pode acontecer pela falta de capacidade de se corrigir o deslocamento do corpo no espaço caso o indivíduo náo gere respostas coordenadas que o tragam de volta ao seu centro de massa corporal ${ }^{34}$.

Além do próprio envelhecimento, é esperado que homens e mulheres idosos com menor atividade física tenham também uma menor massa muscular e, com isso, maior possibilidade de incapacidade física ${ }^{35}$. Neste estudo, o sujeito 1 apresenta diagnóstico de sarcopenia pela diminuição da massa muscular, o que faz que os achados de força e potência muscular estejam consideravelmente diminuídos. Apesar da diminuição de força não ter implicado de forma negativa no controle do equilíbrio corporal, o idoso apresenta histórico de quedas que devem ser evitadas para que náo corra o risco de desenvolver uma maior dependência funcional. Uma vez que a perda de visão nesse caso é permanente, intervençóes para amenizar a perda de massa e força muscular devem focar em exercícios adaptados que visem à redução do número de quedas e aumentem o equilíbrio e a habilidade na marcha ${ }^{36}$. Para tanto, exercícios de resistência muscular devem ser incluídos no programa terapêutico, uma vez que a habilidade para desempenhar atividades da vida diária está bastante relacionada com a massa e a força muscular ${ }^{37}$.

\section{Considerações finais}

Os resultados deste estudo mostraram que o sujeito 1 mesmo sendo sarcopênico e deficiente visual total 
apresentou um bom equilíbrio quando comparado com a média geral e com os sujeitos 2 e 3 . No entanto, nos quesitos avaliados como pico de torque em velocidade de 60 e $180 \%$ seg e trabalho total na velocidade de $180^{\circ} /$ seg, a força muscular está menor em relação aos demais. Este estudo apresentou como limitação a náo realizaçáo de exames complementares a nível visual para verificação de associaçáo de algum dano visual relacionado a DM2, principalmente no sujeito 3 que possui um pior equilíbrio, e a ausência de outro indivíduo deficiente visual total para melhor comparar com os achados do sujeito 1 .

\section{Agradecimentos}

Agradecemos a CAPES pelo apoio da bolsa de estudo (processo: BEX 3113/14-0).

\section{Referências}

1. Rocha OMd, Batista AdAP, Maestá N, Burini RC, Laurindo IMM. Sarcopenia da caquexia reumatoide: conceituação, mecanismos, consequências clínicas e tratamentos possíveis. Rev Bras Reumatol. 2009;49:288-301.

2. Mougios V. Muscle contraction. In: Exercise biochemistry. Illions: Human Kinetics; 2006. p. 105-19.

3. Gomes SCTC. A diabetes mellitus como determinante em saúde e envelhecimento: $\mathrm{O}$ conhecimento do diabético e a presença de complicaçóes da doença [Dissertação]. Lisboa: Universidade Nova de Lisboa; 2011.

4. Pícoli TdS, Figueiredo LLd, Patrizzi LJ. Sarcopenia e envelhecimento. Fisioter. Mov. 2011;24:455-62.

5. Baumgartner RN, Koehler KM, Gallagher D, Romero L, Heymsfield SB, Ross RR, et al. Epidemiology of sarcopenia among the elderly in New Mexico. Am J Epidemiol. 1998; 147(8):755-63.

6. Cruz-Jentoft AJ, Baeyens JP, Bauer JM, Boirie Y, Cederholm T, Landi F, et al. Sarcopenia: European consensus on definition and diagnosis: Report of the European Working Group on Sarcopenia in Older People. Age Ageing. 2010;39(4):412-23.

7. Janssen I, Heymsfield SB, Ross R. Low relative skeletal muscle mass (sarcopenia) in older persons is associated with functional impairment and physical disability. J Am Geriatr Soc. 2002;50(5):889-96.

8. Newman AB, Kupelian V, Visser M, Simonsick E, Goodpaster B, Nevitt M, et al. Sarcopenia: alternative definitions and associations with lower extremity function. J Am Geriatr Soc. 2003;51(11):1602-9.

9. Frisard MI FJ, Russell RD, et al. Physical activity level and physical functionality in nonagenarians compared to individuals aged 60-74 years. J Gerontol A Biol Sci Med Sci. 2007;62(7):783-8.
10. Gazzola JM, Muchale SM, Perracini MR, Cordeiro RC, Ramos LR. Caracterização funcional do equilíbrio de idosos em serviço de reabilitação gerontológica. Rev Fisioter Univ São Paulo. 2004;11(1):1-14.

11. Mirallas NDR, De Conti MHS, De Vitta A, Laurenti R, Saes SdO. Avaliação e reabilitação vestibular no indivíduo idoso. Rev bras geriatr gerontol. 2011;14:687-98.

12. Simoceli L, Bittar RMS, Bottino MA, Bento RF. Perfil diagnóstico do idoso portador de desequilíbrio corporal: resultados preliminares. Rev Bras Otorrinolaringol. 2003;69:772-7.

13. Bittar RSM, Pedalini MEB, Bottino MA, Formigoni LG. Síndrome do desequilíbrio do idoso. Pró-fono. 2002;14(1):119-28.

14. Esquenazi DSS, Guimarães MAM. Aspectos fisiopatológicos do envelhecimento humano e quedas em idosos. Rev HUPE. 2014;13(2):11-20.

15. Maciaszek J, Osinski W, Szeklicki R, Salomon A, Stemplewski R. Body balance parameters established with closed and open eyes in young and elderly men. Biol Sport. 2006;32(2):185-93.

16. Araújo TB, Oliveira RJ, Martins WR, Moura Pereira M, Copetti F, Safons MP. Effects of hippotherapy on mobility, strength and balance in elderly. Arch Gerontol Geriatr. 2013;56(3):478-81.

17. Janssen I, Heymsfield SB, Baumgartner RN, Ross R. Estimation of skeletal muscle mass by bioelectrical impedance analysis. J Appl Physiol. 2000;89(2):465-71.

18. Janssen I, Baumgartner RN, Ross R, Rosenberg IH, Roubenoff R. Skeletal muscle cutpoints associated with elevated physical disability risk in older men and women. Am J Epidemiol. 2004;159(4):413-21.

19. Garcia PA, Dias JMD, Dias RC, Santos P, Zampa CC. Estudo da relação entre função muscular, mobilidade funcional e nível de atividade física em idosos comunitários. Braz J Phys Ther. 2011;15:15-22.

20. Iannuzzi-Sucich M, Prestwood KM, Kenny AM. Prevalence of sarcopenia and predictors of skeletal muscle mass in healthy, older men and women. J Gerontol A Biol Sci Med Sci. 2002;57(12):M772-M7.

21. Morley JE. Diabetes, sarcopenia, and frailty. Clin Geriatr Med. 2008;24(3):455-69.

22. Kim TN, Park MS, Yang SJ, Yoo HJ, Kang HJ, Song W, et al. Prevalence and determinant factors of sarcopenia in patients with type 2 diabetes: the Korean Sarcopenic Obesity Study (KSOS). Diabetes care. 2010;33(7):1497-9.

23. Srikanthan P, Hevener AL, Karlamangla AS. Sarcopenia exacerbates obesity-associated insulin resistance and dysglycemia: findings from the National Health and Nutrition Examination Survey III. PloS one. 2010;5(5):e10805.

24. Williams GN, Higgins MJ, Lewek MD. Aging skeletal muscle: physiologic changes and the effects of training. Phys Ther. 2002;82(1):62-8.

25. Gazzola JM, Perracini MR, Ganança MM, Ganança FF. 
Fatores associados ao equilíbrio funcional em idosos com disfunção vestibular crônica. Rev Bras Otorrinolaringol. 2006;72:683-90.

26. Martins A, Pereira E, Teixeira C, Corazza S. Relação entre força dinâmica máxima de membros inferiores e o equilíbrio corporal em praticantes de musculação. Rev Bras Cineantropom Desempenho Hum. 2010;12(5):375-80.

27. Guimarães L, Galdino D, Martins F, Vitorino D, Pereira K, Carvalho E. Comparação da propensão de quedas entre idosos que praticam atividade física e idosos sedentários. Neurociências. 2004;12(2):68-72.

28. Pedalini M, Alvez N, Bittar RSM, Lorenzi MC, Colello L, Izzo H, et al. Importância de esclarecimentos ministrados em grupo para o equilíbrio do idoso. Arq Int Otorrinolaringol. 2002;6(4):211-6.

29. Freitas EV, et al. Atividade Física no idoso. In: Koogan EG, editor. Tratado de geriatria e gerontologia. 1. ed. Rio de Janeiro; 2002. p. 857-64.

30. Beissner KL, Collins JE, Holmes H. Muscle force and range of motion as predictors of function in older adults. Physical Therapy. 2000;80(6):556-63.
31. Qin W, Yu C. Neural pathways conveying novisual information to the visual cortex. Neural Plast. 2013;14:1-15.

32. Theoret H, Merabet L, Pascual-LeoneA. Behavioral and neuroplastic changes in the blind: evidence for functionally relevant cross-modal interactions. J Physiol. 2004;98(1-3):221-33.

33. Liaw MY, Chen CL, Pei YC, Leong CP, Lau YC. Comparison of the static and dynamic balance performance in young, middle-aged, and elderly healthy people. Chang Gung Med J. 2009;32(3):297-304.

34. Ribeiro AdSB, Pereira JS. Melhora do equilíbrio e redução da possibilidade de queda em idosas após os exercícios de Cawthorne e Cooksey. Rev Bras Otorrinolaringol. 2005;71:38-46.

35. Evans WJ. Effects of exercise on senescent muscle. Clin Ortho Rel Res. 2002;403:S211-S20.

36. Cadore EL, Rodriguez-Manas L, Sinclair A, Izquierdo M. Effects of different exercise interventions on risk of falls, gait ability, and balance in physically frail older adults: a systematic review. Rejuv Res. 2013;16(2):105-14.

37. Doherty TJ. Invited review: aging and sarcopenia. J Appl Physiol. 2003;95:1717-27.

\section{Como citar este artigo:}

Müller DVK, Tavares GMS, Gottlieb MGV, Schneider RH. Equilíbrio e força de um idoso sarcopênico cego: estudo comparativo. Rev. Aten. Saúde. 2016;14(48):54-61. 